\title{
ORIGINAL ARTICLE Phenome-wide analysis of genome-wide polygenic scores
}

\author{
E Krapohl ${ }^{1}$, J Euesden ${ }^{1}$, D Zabaneh ${ }^{1}$, J-B Pingault ${ }^{1,2}$, K Rimfeld $^{1}$, S von Stumm ${ }^{3}$, PS Dale ${ }^{4}$, G Breen ${ }^{1}$, PF O'Reilly ${ }^{1}$ and R Plomin ${ }^{1}$
}

Genome-wide polygenic scores (GPS), which aggregate the effects of thousands of DNA variants from genome-wide association studies (GWAS), have the potential to make genetic predictions for individuals. We conducted a systematic investigation of associations between GPS and many behavioral traits, the behavioral phenome. For 3152 unrelated 16-year-old individuals representative of the United Kingdom, we created 13 GPS from the largest GWAS for psychiatric disorders (for example, schizophrenia, depression and dementia) and cognitive traits (for example, intelligence, educational attainment and intracranial volume). The behavioral phenome included 50 traits from the domains of psychopathology, personality, cognitive abilities and educational achievement. We examined phenome-wide profiles of associations for the entire distribution of each GPS and for the extremes of the GPS distributions. The cognitive GPS yielded stronger predictive power than the psychiatric GPS in our UK-representative sample of adolescents. For example, education GPS explained variation in adolescents' behavior problems $(\sim 0.6 \%)$ and in educational achievement $(\sim 2 \%)$ but psychiatric GPS were associated with neither. Despite the modest effect sizes of current GPS, quantile analyses illustrate the ability to stratify individuals by GPS and opportunities for research. For example, the highest and lowest septiles for the education GPS yielded a 0.5 s.d. difference in mean math grade and a 0.25 s.d. difference in mean behavior problems. We discuss the usefulness and limitations of GPS based on adult GWAS to predict genetic propensities earlier in development.

Molecular Psychiatry (2016) 21, 1188-1193; doi:10.1038/mp.2015.126; published online 25 August 2015

\section{INTRODUCTION}

One of the most striking findings emerging from genome-wide association studies (GWAS) of complex traits is the scarcity of common single nucleotide polymorphism (SNP) associations that account for more than $1 \%$ of trait variation in the population. ${ }^{1,2}$ Although GWAS have been successful in discovering and replicating SNP associations for many traits and disorders, ${ }^{3}$ the dearth of larger SNP associations in well-powered GWAS demonstrates that the ubiquitous heritability of complex dimensions and common disorders is caused by thousands of common DNA variants of small effect. ${ }^{1,4}$ Because their effects are miniscule, a single common SNP is of little use for prediction. For this reason, the future of genetic prediction lies with polygenic scores that aggregate the effects of thousands of SNPs discovered by GWAS, including variants that do not achieve genome-wide significance. ${ }^{5}$ Unlike quantitative genetic designs that estimate the net effect of DNA differences in a population-such as twin and adoption studies and SNP-based heritability ${ }^{6}$ - polygenic scores provide individual-specific estimates of genetic propensities for specific SNPs.

Here we refer to polygenic scores as genome-wide polygenic scores (GPS) for two reasons. First, the acronym GPS excludes the term 'risk', in contrast to the previous labels, which imply that genetic influences are inevitably associated with negative outcomes. Second, the acronym GPS in its original use as global positioning system is an apt metaphor for the use of DNA differences across the genome to guide research on genetic influence.
Association statistics for dozens of large meta-analytic GWAS are now available, including GWAS for psychiatric and cognitive traits. The GPS based on these GWAS results are limited by the 'hidden heritability' ceiling and, as yet, they account for only a few percent of the variance or liability of their target trait. ${ }^{2}$ In addition, most GWAS are based on comparisons between diagnosed cases versus controls using a liability model that assumes continuous liability throughout the population, but the extent to which these case/control results generalize to prediction of continuous traits in the population needs to be established empirically.

Multivariate quantitative genetic analyses using the twin method as well as well as SNP heritability methods have shown that genetic effects are to a substantial extent pleiotropic across complex traits in general $^{7}$ and in particular across cognitive abilities and disabilities ${ }^{8,9}$ and across psychopathologies. ${ }^{10-13}$ This pleiotropy suggests the usefulness of going beyond 'candidate-phenotype' analyses of a single GPS-trait pairing to consider the multivariate profile of GPS associations across many behavioral traits, the behavioral phenome.

Here, we report the first phenome-wide analysis of GPS derived from 13 published major psychiatric, cognitive and biometric GWAS. We applied effect size and significance estimates from GWAS summary statistics to create GPS from raw genotype data for individuals in our target sample. The phenome included 50 traits from the domains of psychopathology, personality, cognitive abilities, and educational achievement, assessed in a representative sample of over 3000 16-year-old individuals in the United Kingdom.

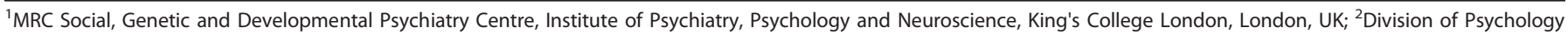

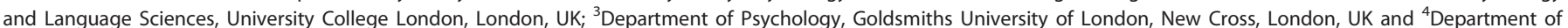

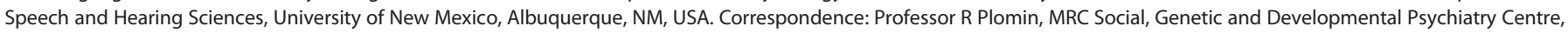
Institute of Psychiatry, Psychology and Neuroscience, King's College London, DeCrespigny Park, Denmark Hill, London SE5 8AF, UK.

E-mail: robert.plomin@kcl.ac.uk

Received 20 May 2015; revised 6 July 2015; accepted 14 July 2015; published online 25 August 2015 
The main focus of this paper is to explore the profile of GPS associations across the behavioral phenome for the entire distribution of each GPS and for the extremes of the GPS distributions. One use of polygenic scores is to predict genetic propensities early in development in order to facilitate interventions that promote potential and prevent problems. As a step in this direction, the present sample consists of adolescents as they finish compulsory schooling at age 16 . We test whether GPS, based on current GWAS, predict phenotypic variation in the adolescent population, and we discuss the usefulness and limitations of GPS based on adult GWAS to predict genetic propensities earlier in development.

\section{MATERIALS AND METHODS}

We used genome-wide genotype and phenome-wide behavioral data from 3152 unrelated adolescents drawn from the UK-representative Twins Early Development Study ${ }^{14-16}$ (Supplementary Table 1). We processed the 3152 genotypes using standard quality control procedures followed by imputation of SNPs using the 1000 Genomes Project reference panel ${ }^{17}$ (Supplementary Methods 1). After quality control, we included around 4.3 million variants into the polygenic score analyses (Supplementary Methods 1). Association analyses were conducted using imputed markers and principal components to control for population stratification. Individuals were assessed on a wide range of phenotypes at the age of 16 . The present analyses included 50 traits from the domains of psychopathology, personality, cognitive abilities and educational achievement (Supplementary Methods 2). All measures were age- and sex-regressed and the $z$-scores were used in the analyses.

We created 13 GPS for each of the over 3000 individuals in our sample using summary statistics from 13 published GWAS ${ }^{18-28}$ (Supplementary Table 2). Here we present results using a $P=0.30$ threshold for including SNPs from the published GWAS (Figure 1 and Supplementary Table 3); results for GPS based on the $P$-value thresholds of 0.10 and 0.05 are included in the Supplementary material (Supplementary Figures $1 \mathrm{a}$ and $\mathrm{b}$ and Supplementary Table 3 ). The selection of the relatively lenient $P=0.30$ threshold was based on the evidence that many associated markers lie within the ensemble of individually non-significant SNPs, with power of the
GPS increasing with number of SNP included. ${ }^{5}$ We also report results (Supplementary Figure 2 and Supplementary Table 4) from a high-resolution polygenic scoring approach, implemented in the software PRSice (London, UK), that identifies the most predictive GPS for each phenotype. ${ }^{29}$

We describe two types of main results: (i) associations between GPS and the behavioral phenome for the entire sample, which demonstrate the usefulness of cross-trait prediction, and (ii) quantile analyses showing the association between selected GPS and behavior by septile, which illustrates the ability to stratify individuals by GPS and the potential of polygenic score for phenotype prediction.

To inform these analyses, we demonstrate that GPS are normally distributed and discuss the implications for considering both endsresilience as well as risk-of GPS distributions. We also examine three types of correlations: (i) genetic correlations between the GWAS summary statistics (ii) correlations between the GPS, and (iii) phenotypic correlations between the target phenotypes. These correlations support the usefulness of a phenome-wide analysis of GPS.

\section{RESULTS}

GPS are normally distributed

The quantitative genetic model assumes that many genetic variants of small effect drive the heritability of complex traits and common disorders, ${ }^{30}$ even though each marker is inherited in the discrete manner hypothesized by Mendel. ${ }^{31}$ Therefore, the central limit theorem implies that the distribution of polygenic scores in the population will approach normality. Specifically, the normal distribution is to be expected whenever trait variation is polygenic and produced by the addition of a large number of small effects.

Nonetheless, the normality of GPS (Supplementary Figures 3a and c) merits emphasis because it illustrates that common disorders can be considered as extremes of the common polygenic liability spectrum, which has far-reaching implications for diagnosis, treatment and prevention. ${ }^{32}$ It also implies that GPS can be operationalized in terms of 'resilience' as well as 'risk' predictors. There is untapped research potential for operationalizing the

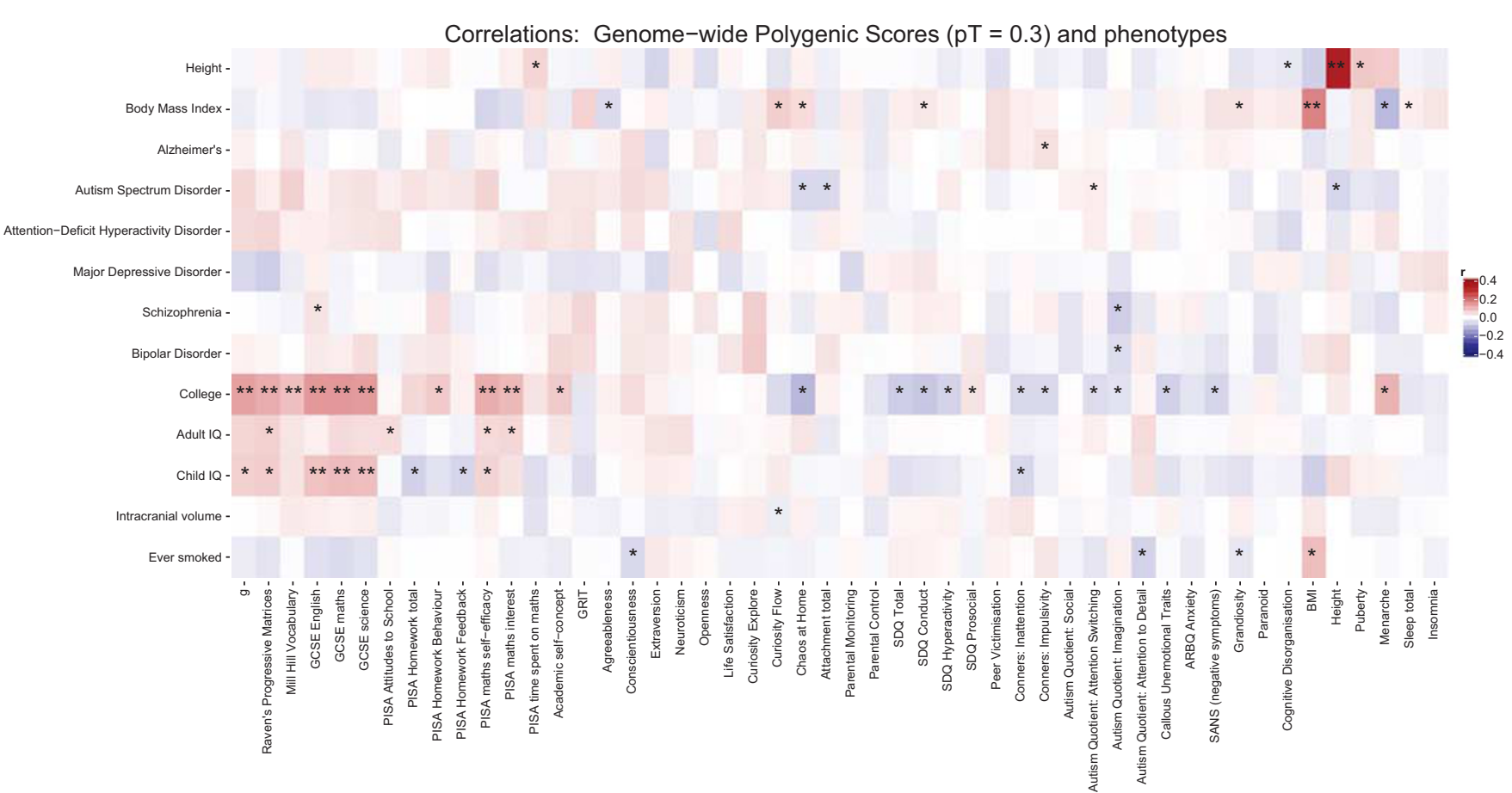

Figure 1. Correlations between 13 genome-wide polygenic scores and 50 traits from the behavioral phenome. These results are based on GPS constructed using a GWAS $P$-value threshold $\left(P_{\mathrm{T}}\right)=0.30$; results for $P_{\mathrm{T}}=0.10$ and 0.05 (Supplementary Figures $1 \mathrm{a}$ and $\mathrm{b}$ and Supplementary Table 3). $P$-values that pass Nyholt-Sidak correction (see Supplementary Methods 1 ) are indicated with two asterisks, whereas those reaching nominal significance (thus suggestive evidence) are shown with a single asterisk. 
negative tail of GPS for disorders as 'resilience' and the negative end of cognitive or education GPS as 'risk' factors. This 'other end' of the normal distribution of GPS is uncharted territory. From an evolutionary perspective, averageness might be an adaptive tradeoff against the mishmash of costs and benefits of more extreme GPS, especially given the fluctuating nature of selection. ${ }^{32}$

Intercorrelations between GWAS, GPS and phenotypic traits

As depicted in Supplementary Figure 4 the phenotypic correlations between the target phenotypes in our sample of adolescents show substantial intercorrelations, with a 'cognitive' and a 'psychopathology' cluster.

We estimate the genetic correlation between the discovery GWAS using a new technique based on LD score regression, ${ }^{33,34}$ which uses only GWAS summary statistics and linkage disequilibrium information to decompose true polygenic variance/ covariance from confounding (see Supplementary Methods for details). Supplementary Figure 5 depicts the genetic correlations between the 13 GWAS, which provide evidence for significant and substantial pleiotropy. In addition to the genetic correlations reported previously, ${ }^{34}$ we add correlations for the summary statistics of the child IQ GWAS, ${ }^{19}$ adult $I^{35}$ and intracranial volume. $^{20}$ The observed genetic correlations replicate and extend previous research. We confirm genetic overlap between the major psychoses $^{13,25,34,36,37}$ and between cognitive phenotypes including intracranial volume, ${ }^{18,20,38-40}$ respectively. We further find correlations between these two clusters-for example, strong negative associations between the cognitive phenotypes and Alzheimer's and positive associations between educational attainment and autism spectrum disorder as well as bipolar disorder.

We also examined correlations between the GPS created for our sample (Supplementary Figures $6 a$ and $c$ and Supplementary Table 3). We find similar correlation patterns but weaker overall correlations.

These genetic correlations provide evidence that polygenic effects are to a substantial degree pleiotropic across traits. Together with finding substantial correlations between the target phenotypes, this multivariate genetic architecture suggests the usefulness of a phenome-wide approach to investigate the links between GPS and behavior, which is the focus of the next and final section of results.

\section{GPS correlate with the behavioral phenome}

Figure 1 summarizes correlations between the 50 traits of the behavioral phenome and the $13 \mathrm{GPS}$ for $P_{\mathrm{T}}=0.30$. Correlation coefficients, s.e., $P$-value thresholds $\left(P_{\mathrm{T}}\right)$, and number of SNPs included are shown in Supplementary Table 3 for the fixed $P_{\mathrm{T}}(0.30 ; 0.10 ; 0.05)$. Very similar patterns of association emerged from both the conventional fixed $P_{\mathrm{T}}$ analyses and the highresolution analyses that estimate the $P_{\mathrm{T}}$ flexibly for the 'best-fit' GPS (Supplementary Figure 2 and Supplementary Table 4). Both methods yielded statistically significant phenomic associations only for the GPS for College and Child IQ.

College GPS. College GPS, which was based on the binary measure of attending college or not, showed the strongest phenomic profile at age 16, which might reflect the fact that its meta-analytic GWAS sample size was one of the largest $\left(N=120000\right.$; Rietveld et al. $\left.{ }^{18}\right)$. College GPS correlated significantly with academic performance at age 16: General Certificate of Secondary Examination (GCSE) English $(r=0.15)$, GCSE mathematics $(r=0.15$, s.e. 0.02$)$ and GCSE science $(r=0.14$, s.e. 0.02$){ }^{39}$ College GPS also correlated significantly with general cognitive ability $\left({ }^{\prime} \mathrm{g}\right.$ ') $(r=0.14$, s.e. 0.03$)$ as well as its subscales Ravens Matrices $(r=0.12$, s.e. 0.03$)$ and with Mill Hill Vocabulary $(r=0.09$, s.e. 0.03$)$, which confirms a similar finding for adults. ${ }^{40}$ College GPS also correlated positively with PISA math interest $(r=0.10$, s.e. 0.03$)$ and math self-efficacy ( $r=0.12$, s.e. 0.03 ). Negative associations for College GPS emerged for SDQ total behavior problems ( $r=-0.07$, s.e. 0.02$)$ and SDQ Conduct ( $r=-0.08$, s.e. 0.02$)$.

Child IQ GPS. The GPS for Child IQ yielded a similar but diluted phenomic profile as compared with College GPS. Child IQ GPS correlated significantly with GCSE English ( $r=0.09$, s.e. $0.02)$, GCSE Math ( $r=0.10$, s.e. 0.02$)$ and GCSE Science $(r=0.09$, s.e. 0.02 ).

Psychiatric GPS. In contrast, the five psychiatric GPS yielded no significant correlations that passed multiple comparisons corrections across the behavioral phenome. Nominally significant associations included a positive correlation between Alzheimer's GPS and Conner's Impulsivity; and positive associations between Autism Spectrum Disorder GPS and Autism Quotient: Attention Switching. Autism Spectrum Disorder GPS yielded nominally significant negative associations with Chaos at home, Attachment and Height. Schizophrenia GPS correlated positively with GCSE English and negatively with Autism Quotient: Attention to Detail. Bipolar disorder GPS correlated negatively with Autism Quotient: Attention to Detail.

One likely explanation for the lower phenomic profile of psychiatric GPS compared with that of College GPS is the difference in sample sizes for the discovery samples. However, Child IQ GPS yielded significant associations despite the relatively smaller sample size of the GWAS $(N=9616)$. This might point to the importance of developmental proximity or similarity of the phenotypes in discovery and target sample. It also emphasizes that predictive power is not only a function of sample size of the discovery sample. ${ }^{5}$ Phenotypic similarity between the traits in the discovery sample and the target sample is a proxy for the magnitude of genetic covariance between the traits.

The underlying premise of GWAS is that the polygenic architecture of complex traits and common disorders requires a genome-wide approach despite the multiple testing burden. Similarly, based on strong evidence for the ubiquitous pleiotropy of complex traits, ${ }^{7,9-13,34}$ the advantage of the phenome-wide approach outweighs the resulting multiple testing burden. Specifically, while testing a large number of highly unlikely hypotheses with little or no prior support should be avoided, in this case we have collated a well-defined set of psychological and behavioral traits for which there is good reason to suspect causal associations with the available discovery GWAS phenotypes. In this way, the only 'multiple testing problem' relates to setting an appropriate significance threshold given the number and correlation of tests performed (see Supplementary Methods for multiple testing correction method used).

Therefore, the absence of phenome-wide significant associations (that is, after correcting for multiple testing across the 50 traits and 13 GPS) for all psychiatric GPS does not imply the absence of polygenic effects. However, the scarcity of nominally significant associations between the psychiatric GPS and the 50 traits suggests that the genetic covariance between psychiatric adult case/control samples and our adolescent population sample might be relatively small. For instance, under certain assumptions about polygenic architecture (for example, $\leqslant 5 \%$ of tested SNPs associated with schizophrenia in the discovery GWAS), we had $\geqslant 80 \%$ power with $a=0.05$ to detect associations between the Schizophrenia GPS and a phenotype given $\geqslant 0.06$ genetic covariance between schizophrenia and the target trait, with $\geqslant 0.5 \%$ of phenotypic variation in the target trait explained by schizophrenia ${ }^{5,41,42}$ (see Supplementary Methods for more detail).

One possible reason for the lower observed phenomic profile of the psychiatric GPS might be that the current sample is UK representative and therefore not enriched for psychiatric symptoms. The psychiatric GPS were based on case-control comparisons, often with extreme cases. This emphasizes the limitations of using GPS for 
a

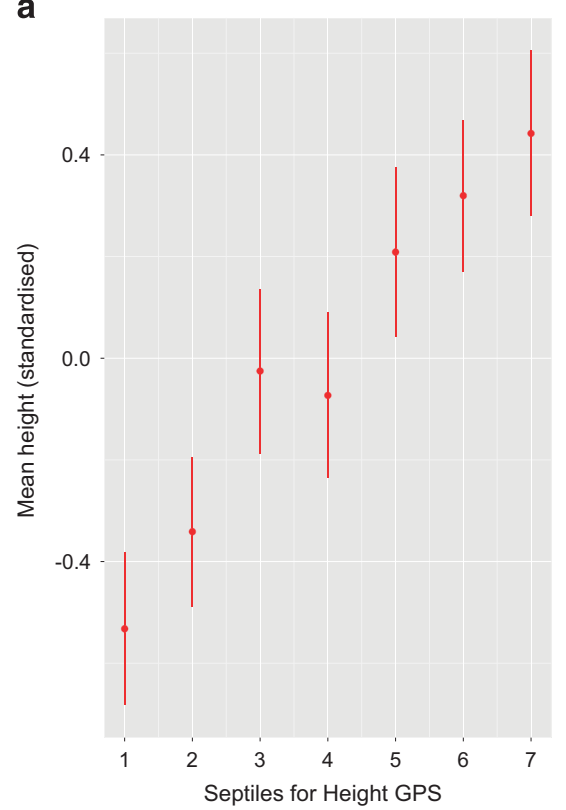

b

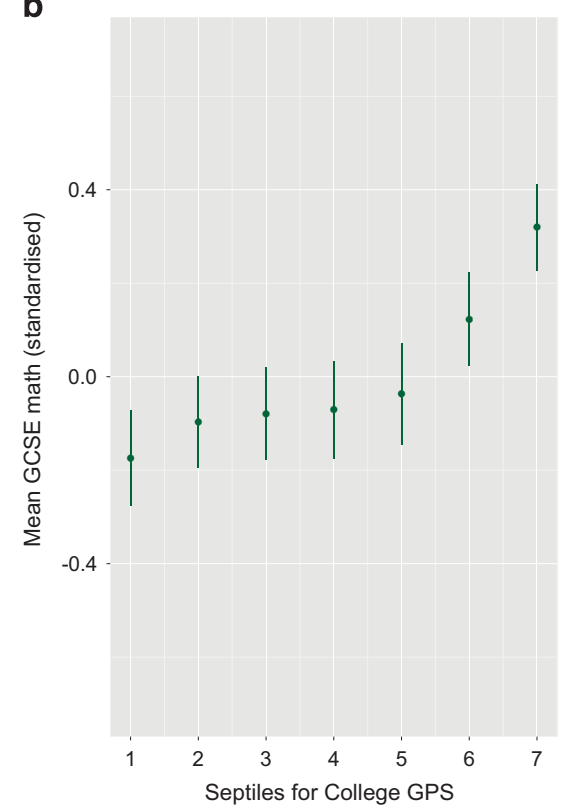

C

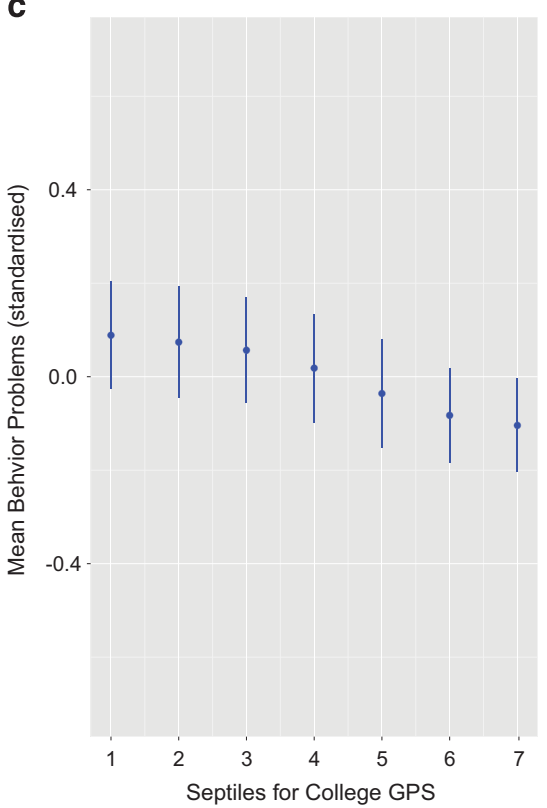

Figure 2. (a) Mean for height at age 16 by adult Height genome-wide polygenic score (GPS) septile. The threshold for selecting trait-associated alleles was $P_{\mathrm{T}}<0.30$. The GPS were converted to quantiles $(1=$ lowest, $7=$ highest GPS). Mean phenotypic values and $95 \%$ confidence intervals $(\mathrm{Cls})$ for the quantile groups (bars) were estimated using general linear regression with ancestrally informative principal components, sex and age of measurement as covariates. (b) Mean for children's mathematics educational achievement at age 16 (compulsory subject on the General Certificate of Secondary Examination (GCSE), see Materials and Methods for details) by College GPS septile. The threshold for selecting trait-associated alleles was $P_{\mathrm{T}}<0.30$. The GPS were converted to quantiles $(1=$ lowest, $7=$ highest GPS). Mean phenotypic values and $95 \% \mathrm{Cl}$ for the quantile groups (bars) were estimated using general linear regression with ancestrally informative principal components, sex and age of measurement as covariates. (c) Mean for total parent-reported behavior problems at age 16 by adult College GPS septile. The threshold for selecting trait-associated alleles was $P_{\mathrm{T}}<0.30$ (the best-fit GPS as estimated by PRSice software, see Materials and Methods). The GPS were converted to quantiles ( 1 =lowest, $7=$ highest GPS). Mean phenotypic values and $95 \% \mathrm{Cl}$ for the quantile groups (bars) were estimated using general linear regression with ancestrally informative principal components, sex and age of measurement as covariates.

the prediction of trait variation in the general population from GWAS based on selected samples. Importantly, the GPS College did predict children's behavior problems in our UK-representative sample, whereas the psychiatric GPS did not. This points to the usefulness of cross-trait prediction in general and the value of cognitive GWAS/GPS as prediction instruments for psychiatric symptoms in the population.

Other GPS. Adult body mass index (BMI) GPS correlate positively with the measure of BMI at age $16(r=0.18$, s.e. 0.03$)$; and adult Height GPS correlate with height at age $16(r=0.33$, s.e. 0.03$)$. There was suggestive evidence for a negative association between Ever smoked GPS and conscientiousness $(r=-0.06$, s.e. 0.03$)$ and a positive association with BMI $(r=0.09$, s.e. 0.03$)$.

\section{Quantile analyses}

To illustrate the ability to stratify individuals by GPS and the potential of polygenic score for phenotype prediction, we grouped individuals into GPS septiles and estimated the mean phenotypic value for each quantile. We provide three examples:

Figure $2 a$ shows that mean standardized height increased with more adult height-associated alleles in our UK-representative sample of children aged 16, with the largest difference between the lowest and highest septile (Hedges $g$ : -1.01 with $95 \%$ confidence interval $(\mathrm{Cl}):-1.26$ to -0.77 ; difference in means: 0.97 s.d., with $P$-value $<0.01$ ). Figure $2 \mathrm{~b}$ shows that mean math grade on the standardized UK-national examinations at age 16 increased with more Collegeassociated alleles (Figure 2b), with the largest difference between the lowest and highest septile (Hedges $g$ : -0.52 with $95 \% \mathrm{Cl}:-0.67$ to -0.37 ; difference in means: 0.49 s.d., with $P$-value $<0.01$ ).
Figure $2 c$ illustrates the utility of the phenome-wide approach for cross-trait prediction: the mean for total parent-reported behavior problems at age 16 decreased slightly but significantly with higher College GPS, with a maximum effect size between the lowest and highest quantile (Hedges $g$ : 0.20 with $95 \% \mathrm{Cl}$ : 0.04-0.34; difference in means: 0.19 , with $P$-value 0.01).

These results (Figure 2) illustrate the ability to stratify individuals by GPS, which suggests opportunities for research, for example, selecting high and low GPS extreme individuals for intensive research such as neuroimaging that is unable to test large representative samples. However, we emphasize that the current predictive power and accuracy of GPS do not allow for their use as predictive tests.

\section{DISCUSSION}

These results highlight the usefulness of a phenome-wide approach to examine behavioral profiles of associations with GPS even though current GPS account for only a few percent of variance or liability of their target trait. An interesting finding is that phenome-wide associations for cognitive GPS are stronger than for psychiatric GPS in our UK-representative sample of adolescents. For example, we found that GPS College, but none of the psychiatric GPS, predicted adolescent behavior problems, which demonstrates the usefulness of cross-trait predictions and the multivariate phenome-wide approach in general. However, this finding could be explained by differences in sample sizes, sampling methods (population versus case/control), and genetic architecture (for example, extent of covariance between discovery and target trait). 
Finding significant associations for the Child IQ GPS, which is based on a small discovery sample, is a reminder that predictive power of GPS is not merely a function of sample size but also of the developmental proximity of the GWAS sample and the target GPS sample. As explained in the Introduction, we were interested in the extent to which GWAS in adult samples yield GPS that can predict genetic propensities - strengths as well as weaknessesearlier in development, in this case in adolescence. However, GPS College is a trait assessed closer in age to the adolescents in our sample. In contrast, the psychiatric GPS were derived from GWAS studies of adults.

A larger issue is that extant GPS account for only a few percent of the phenomic variance in the target trait. However, we illustrate the research potential of polygenic stratification by quantile. Power and accuracy of GPS will improve as GWAS sample sizes increase. GPS that narrow the 'hidden heritability' gap is what is needed most for phenome-wide analyses-and for all research harvesting the fruits of GWAS.

\section{CONFLICT OF INTEREST}

The authors declare no conflict of interest.

\section{ACKNOWLEDGMENTS}

We gratefully acknowledge the ongoing contribution of the participants in the Twins Early Development Study (TEDS) and their families. TEDS is supported by a program grant to RP from the UK Medical Research Council (G0901245; and previously G0500079), with additional support from the US National Institutes of Health (HD044454; HD059215). EK is supported by a Medical Research Council studentship. RP is supported by a Medical Research Council Research Professorship award (G19/2) and a European Research Council Advanced Investigator award (295366). GB was funded in part by the National Institute for Health Research Biomedical Research Centre for Mental Health at South London and Maudsley NHS Foundation Trust and the Institute of Psychiatry, Psychology \& Neuroscience, King's College London.

\section{AUTHOR CONTRIBUTIONS}

$\mathrm{RP}$ directs and received funding for the Twins Early Development Study (TEDS). $\mathrm{RP}$ and EK conceived of the present study. EK analyzed and interpreted the data. RP and PFO supervised the project and interpreted the data. RP and EK wrote the manuscript with help from PFO, DZ, J-BP, KR, SVS, PSD and GB. JE provided an early version of the PRSice software (http://PRSice.info/).

\section{REFERENCES}

1 Gratten J, Wray NR, Keller MC, Visscher PM. Large-scale genomics unveils the genetic architecture of psychiatric disorders. Nat Neurosci 2014; 17: 782-790.

2 Wray NR, Lee SH, Mehta D, Vinkhuyzen AAE, Dudbridge F, Middeldorp CM. Research review: polygenic methods and their application to psychiatric traits. $J$ Child Psychol Psychiatry 2014; 55: 1068-1087.

3 Visscher PM, Brown MA, McCarthy MI, Yang J. Five years of GWAS discovery. Am J Hum Genet 2012; 90: 7-24.

4 Robinson MR, Wray NR, Visscher PM. Explaining additional genetic variation in complex traits. Trends Genet 2014; 30: 124-132.

5 Dudbridge F. Power and predictive accuracy of polygenic risk scores. PLoS Genet 2013; 9: e1003348.

6 Yang JA, Lee SH, Goddard ME, Visscher PM. GCTA: a tool for genome-wide complex trait analysis. Am J Hum Genet 2011; 88: 76-82.

7 Sivakumaran S, Agakov F, Theodoratou E, Prendergast JG, Zgaga L, Manolio T et al. Abundant pleiotropy in human complex diseases and traits. Am J Hum Genet 2011; 89: 607-618.

8 Plomin R, Kovas Y. Generalist genes and learning disabilities. Psychol Bull 2005; 131: 592-617.

9 Trzaskowski M, Davis OSP, DeFries JC, Yang J, Visscher PM, Plomin R. DNA evidence for strong genome-wide pleiotropy of cognitive and learning abilities. Behav Genet 2013; 43: 267-273.

10 Kendler KS, Aggen SH, Knudsen GP, Røysamb E, Neale MC, Reichborn-Kjennerud T. The structure of genetic and environmental risk factors for syndromal and subsyndromal common DSM-IV Axis I and All Axis II disorders. Am J Psychiatry 2011; 168: 29-39.
11 Lichtenstein P, Carlström E, Råstam M, Gillberg C, Anckarsäter H. The genetics of autism spectrum disorders and related neuropsychiatric disorders in childhood. Am J Psychiatry 2010; 167: 1357-1363.

12 Lichtenstein P, Yip BH, Björk C, Pawitan Y, Cannon TD, Sullivan PF et al. Common genetic determinants of schizophrenia and bipolar disorder in Swedish families: a population-based study. Lancet 2009; 373: 234-239.

13 Cross-Disorder Group of the Psychiatric Genomics Consortium. Genetic relationship between five psychiatric disorders estimated from genome-wide SNPs. Nat Genet 2013; 45: 984-994.

14 Haworth CMA, Davis OSP, Plomin R. Twins Early Development Study (TEDS): a genetically sensitive investigation of cognitive and behavioral development from childhood to young adulthood. Twin Res Hum Genet 2013; 16: 117-125.

15 Oliver BR, Plomin R. Twins' Early Development Study (TEDS): a multivariate, longitudinal genetic investigation of language, cognition and behavior problems from childhood through adolescence. Twin Res Hum Genet 2007; 10: 96-105.

16 Kovas Y, Haworth CMA, Dale PS, Plomin R. The genetic and environmental origins of learning abilities and disabilities in the early school years. Monogr Soc Res Child Dev 2007; 72, vii 1-144.

17 Abecasis GR, Auton A, Brooks LD, DePristo MA, Durbin RM, Handsaker RE et al. An integrated map of genetic variation from 1,092 human genomes. Nature 2012; 491: 56-65.

18 Rietveld CA, Medland SE, Derringer J, Yang J, Esko T, Martin NW et al. GWAS of 126,559 individuals identifies genetic variants associated with educational attainment. Science 2013; 340: 1467-1471.

19 Benyamin B, Pourcain BS, Davis OS, Davies G, Hansell NK, Brion M-J et al. Childhood intelligence is heritable, highly polygenic and associated with FNBP1L. Mol Psychiatry 2014; 19: 253-258.

20 Hibar DP, Stein JL, Renteria ME, Arias-Vasquez A, Desrivières S, Jahanshad N et al. Common genetic variants influence human subcortical brain structures. Nature 2015; 520: 224-229.

21 Lambert JC, Ibrahim-Verbaas CA, Harold D, Naj AC, Sims R, Bellenguez C et al. Meta-analysis of 74,046 individuals identifies 11 new susceptibility loci for Alzheimer's disease. Nat Genet 2013; 45: 1452-1458.

22 Psychiatric GWAS Consortium Bipolar Disorder Working Group. Large-scale genome-wide association analysis of bipolar disorder identifies a new susceptibility locus near ODZ4. Nat Genet 2011; 43: 977-983.

23 Major Depressive Disorder Working Group of the Psychiatric GWAS Consortium, Ripke S, Wray NR, Lewis CM, Hamilton SP, Weissman MM et al. A mega-analysis of genome-wide association studies for major depressive disorder. Mol Psychiatry 2013; 18: 497-511.

24 Schizophrenia Working Group of the Psychiatric Genomics Consortium. Biological insights from 108 schizophrenia-associated genetic loci. Nature 2014; 511: 421-427.

25 Cross-Disorder Group of the Psychiatric Genomics Consortium. Identification of risk loci with shared effects on five major psychiatric disorders: a genome-wide analysis. Lancet 2013; 381: 1371-1379.

26 Wood AR, Esko T, Yang J, Vedantam S, Pers TH, Gustafsson S et al.Defining the role of common variation in the genomic and biological architecture of adult human height. Nat Genet 2014; 46: 1173-1186.

27 Tobacco and Genetics Consortium. Genome-wide meta-analyses identify multiple loci associated with smoking behavior. Nat Genet 2010; 42: 441-447.

28 Locke AE, Kahali B, Berndt SI, Justice AE, Pers TH, Day FR et al.Genetic studies of body mass index yield new insights for obesity biology. Nature 2015; 518: 197-206.

29 Euesden J, Lewis CM, O'Reilly PF. PRSice: polygenic risk score software. Bioinformatics 2015; 31: 1466-1468.

30 Fisher RA. The correlation between relatives on the supposition of Mendelian inheritance. Transactions of the Royal Society of Edinburgh 1918; 52: 399-433.

31 Mendel G. Versuche über Pflanzenhybriden. Verhandlungen Naturforschenden Vereines Brunn 1866; 4: 3-47.

32 Plomin R, Haworth CMA, Davis OSP. Common disorders are quantitative traits. Nat Rev Genet 2009; 10: 872-878.

33 Bulik-Sullivan BK, Loh P-R, Finucane HK, Ripke S, Yang J, Schizophrenia Working Group of the Psychiatric Genomics Consortium et al. LD score regression distinguishes confounding from polygenicity in genome-wide association studies. Nat Genet 2015; 47: 291-295.

34 Bulik-Sullivan B, Finucane HK, Anttila V, Gusev A, Day FR, ReproGen Consortium et al. An atlas of genetic correlations across human diseases and traits. bioRxiv 2015; 014498.

35 Davies G, Armstrong N, Bis JC, Bressler J, Chouraki V, Giddaluru S et al. Genetic contributions to variation in general cognitive function: a meta-analysis of genome-wide association studies in the CHARGE consortium ( $\mathrm{N}=53949$ ). Mol Psychiatry 2015; 20: 183-192.

36 Kavanagh DH, Tansey KE, O'Donovan MC, Owen MJ. Schizophrenia genetics: emerging themes for a complex disorder. Mol Psychiatry 2015; 20: 72-76. 
37 Maier R, Moser G, Chen G-B, Ripke S, Cross-Disorder Working Group of the Psychiatric Genomics Consortium, Coryell W et al. Joint analysis of psychiatric disorders increases accuracy of risk prediction for schizophrenia, bipolar disorder, and major depressive disorder. Am J Hum Genet 2015; 96: 283-294.

38 Trzaskowski M, Harlaar N, Arden R, Krapohl E, Rimfeld K, McMillan A et al. Genetic influence on family socioeconomic status and children's intelligence. Intelligence 2014; 42: 83-88.

39 Krapohl E, Plomin R. Genetic link between family socioeconomic status and children's educational achievement estimated from genome-wide SNPs. Mol Psychiatry advance online publication, 10 March 2015; doi:10.1038/mp.2015.2.

40 Rietveld CA, Esko T, Davies G, Pers TH, Turley P, Benyamin B et al. Common genetic variants associated with cognitive performance identified using the proxy-phenotype method. Proc Natl Acad Sci USA 2014; 111: 13790-13794.

41 Wray NR, Goddard ME, Visscher PM. Prediction of individual genetic risk to disease from genome-wide association studies. Genome Res 2007; 17: 1520-1528.
42 Chatterjee N, Wheeler B, Sampson J, Hartge P, Chanock SJ, Park J-H. Projecting the performance of risk prediction based on polygenic analyses of genome-wide association studies. Nat Genet 2013; 45: 400-405.

(i) This work is licensed under a Creative Commons Attribution 4.0 International License. The images or other third party material in this article are included in the article's Creative Commons license, unless indicated otherwise in the credit line; if the material is not included under the Creative Commons license, users will need to obtain permission from the license holder to reproduce the material. To view a copy of this license, visit http://creativecommons.org/licenses/ by/4.0/

(c) The Author(s) 2016

Supplementary Information accompanies the paper on the Molecular Psychiatry website (http://www.nature.com/mp) 